\title{
Cooperativity in ionic liquids
}

\author{
Simone Koßmann, Jens Thar, and Barbara Kirchner ${ }^{\text {a) }}$ \\ Institut für Physikalische und Theoretische Chemie, Universität Bonn, Wegelerstrasse 12, D-53115 Bonn, \\ Germany
}

\author{
Patricia A. Hunt and Tom Welton \\ Department of Chemistry, Imperial College of Science, Technology and Medicine, London SW7 2BY, \\ United Kingdom
}

(Received 8 December 2005; accepted 9 March 2006; published online 2 May 2006)

\begin{abstract}
Cooperativity in ionic liquids is investigated by means of static quantum chemical calculations. Larger clusters of the dimethylimidazolium cation paired with a chloride anion are calculated within density functional theory combined with gradient corrected functionals. Tests of the monomer unit show that density functional theory performs reasonably well. Linear chain and ring aggregates have been considered and geometries are found to be comparable with liquid phase structures. Cooperative effects occur when the total energy of the oligomer differs from a simple sum of monomer energies. Cooperative effects have been found in the structural motifs examined. A systematic study of linear chains of increasing length (up to nine monomer units) has shown that cooperativity plays a more important role than expected and is stronger than in water. The $\mathrm{Cl} \cdots \mathrm{H}$ distance of the chloride to the most acidic proton increases with an increasing number of monomer units. The average bond distance approaches $218.9 \mathrm{pm}$ asymptotically. The dipole moment grows almost linearly and the dipole moment per monomer unit reaches the asymptotic value of $16.3 \mathrm{D}$. The charge on the chloride atoms decreases with an increasing chain length. In order to detect local hydrogen bonding in the clusters a new parametrization of the shared-electron number method is introduced. We find decreasing hydrogen bond energies with an increasing cluster size for both the first hydrogen bond to the most acidic proton and the average hydrogen bond. () 2006 American Institute of Physics. [DOI: 10.1063/1.2191493]
\end{abstract}

\section{INTRODUCTION}

The wide applicability of ionic liquids as environmentfriendly solvents has recently led to a steep increase in publications on this subject. Ionic liquids are fused salts of organic cations and most often inorganic anions. ${ }^{1,2}$ They are good solvents for a wide range of both inorganic and organic reactions. The solvating ability of ionic liquids is generally attributed to their high polarity and noncoordinating behavior. ${ }^{1,2}$ However, the details of these interactions are still not well understood.

Despite the wide interest in ionic liquids, and the microscopic behavior of these liquids being accessible by means of theoretical calculations, a few theoretical studies on the subject have appeared in the literature. Static electronic structure calculations have mainly been restricted to density functional theory, Hartree-Fock, MP2, and semiempirical calculations. Furthermore, in many cases these calculations have been restricted to a single ion or ionic pair (consisting of one anion and one cation). ${ }^{3-16}$ In several studies a number of ion-pair isomers have been examined. Turner et al. have investigated a number of small 1-alkyl-3-methylimidazolium cations (alkyl chain $R=$ methyl, ethyl, and butyl) associated with halides spanning $\mathrm{F}$ to $\mathrm{I} .{ }^{17}$ The interaction energies of 1-ethyl3-methylimidazolium cations combined with one to three halide anions have been investigated by Wang et al. ${ }^{18}$ Hunt and

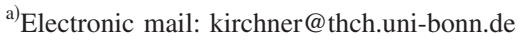

Gould have analyzed in a very excellent article the relative energy and halide ion position for isomers of 1-butyl-3methylimidazolium paired with a chloride anion. ${ }^{19}$ Within these articles a good overview of recent calculations is presented. None of the above cited works treats several pairs. Therefore these calculations are certainly not sufficient enough to model the condensed phase.

A superior approach to the condensed phase is given by molecular dynamics simulations. Traditional molecular dynamics simulations of ionic liquids employing pair potentials were pioneered by Hanke et al. ${ }^{20,21}$ and Lynden-Bell. ${ }^{22}$ Polarizable force fields have also been tested for molecular dynamics simulations of ionic liquids. ${ }^{23}$ So far, only three first-principle simulations have appeared in literature; these have all examined the 1,3-dimethylimidazolium chloride $\left(\left[\mathrm{C}_{1} \mathrm{C}_{1} i m\right] \mathrm{Cl}\right)$ ionic liquid. ${ }^{24-26}$ First-principle simulations have the advantage that the electronic structure is calculated on the fly. Therefore, polarizable effects are treated implicitly. In Ref. 24 Car-Parrinello molecular dynamics simulations with 25 pairs and 41 pairs, both with an energy cutoff of $60 \mathrm{Ry}$, were undertaken. Reference 25 presents a simulation with 8 pairs ( $39 \mathrm{ps})$ and one with 24 pairs $(3.5 \mathrm{ps})$ of the $\left[\mathrm{C}_{1} \mathrm{C}_{1}\right.$ im $] \mathrm{Cl}$ system. A 32 ion-pair Car-Parrinello simulation at $425 \mathrm{~K}$ run for almost $10 \mathrm{ps}$ was undertaken by Bhargava and Balasubramanian. ${ }^{26}$

In this study we bridge the gap between static electronic structure calculations and classical molecular dynamics 
simulations in that we concentrate on static quantum chemical calculations of larger clusters built from one pair of the dimethylimidazolium cation $\left(\left[\mathrm{C}_{1} \mathrm{C}_{1} \mathrm{im}\right]^{+}\right)$combined with the chloride anion. The main focus of this article aims at understanding cooperative effects in ionic liquids.

Cooperative or many-body effects can be defined as follows: If for any property the sum of the value for the monomeric unit (single particle property) is added up $m$ times and deviates from the value for the $m$ oligomer, then this behavior is nonadditive and the difference shall be termed cooperative or many-body effect. For instance, the binding energy of a $m$ oligomer displays cooperativity if this energy is greater than the sum of $m$ times the dimer binding energy and anticooperativity if the binding energy is less than this sum. For the binding energy the lowest particle property is between two particles.

Since, for cooperativity considerations, more than one species must be involved, the investigation of cooperativity or many-body effects in condensed phase is especially interesting. In standard simulations the forces on particles are usually derived from the pairwise additivity ansatz. This approximation assumes that the total force on a particle can be derived from adding up the forces between the particle and all its neighbors. Depending on the chemical system such a force can be nonadditive, i.e., it cannot be calculated as a sum of two-body contributions, but requires additional cooperative or many-body terms. Contrary to relativistic effects, the investigation of cooperative effects cannot be easily achieved by exchanging the "wrong" equation by the "right" equation, i.e., by exchanging the Schrödinger equation by the Dirac equation. It is not trivial to add systematically manybody terms to simulations when the aim is to extract cooperative effects. Adding many-body forces to a pure two-body force was so far only been done systematically (i.e., a calculated pure two-body potential and higher-order potentials) for rare gas systems. The parametrization of a three-body potential is still a demanding task but at least it is possible to carry out this parametrization. ${ }^{27,28}$ The parametrization of a three-body potential for water where all degrees of freedom are considered has, as far as we know, not been undertaken so far and probably will not be undertaken in the foreseeable future, because of the high dimensionality of the problem. In the work of van der Avoird et al. a three-body potential for water was calculated where only three protons are allowed to move. ${ }^{29}$ Polarizable force fields do improve simulations substantially. Nevertheless, they do not systematically extract cooperative effects, because empirical potentials usually already aim at reproducing the right thermodynamic property and are therefore effective but not pure two-body potentials. Using a first-principle ansatz in simulations where the total forces are derived from the total electronic structure to achieve this goal is not simple, since it is not trivial to restrict the electronic structure calculations only to pairs. ${ }^{30}$ For more complicated systems, i.e., for systems that are not chemically inert such as rare gases, a systematic study can be undertaken by means of static cluster calculations. This neglects temperature and dynamic effects, but gives an estimate of the electronically induced cooperative effects in complicated systems, such as ionic liquids. While the cooperative charac- ter of hydrogen bonding is under intensive investigation, ${ }^{31-35}$ no such study has explored the cooperative nature of ionic liquids.

The next section describes the methodology used. This is followed by the results part where first the monomer unit is introduced. After this we shed some light onto dimer and trimer configurations. We proceed in the results part with a systematic assessment of cooperative effects in larger clusters with up to nine units. Finally, conclusions and discussions end this article.

\section{METHODOLOGY}

For the quantum chemical structure optimizations we use the density functional and $a b$ initio programs provided by the TURBOMOLE 5.1 suite. ${ }^{36}$ We employ the gradient corrected functional BP86 (Refs. 37 and 38) with the resolution of the identity (RI) technique ${ }^{36}$ and the hybrid functional B3LYP. ${ }^{39,40}$ Moreover, we apply second-order Møller-Plesset perturbation theory (MP2) in combination with the RI technique. ${ }^{36}$ The density functional theory (DFT) results are obtained from all-electron restricted Kohn-Sham calculations. Ahlrichs' TZVP basis set is used throughout featuring a valence triple-zeta basis set with polarization functions on all atoms unless otherwise stated. ${ }^{41}$ For some structures we also test the larger basis set TZVPP (Ref. 36) and the Dunning basis sets aug-cc-pVDZ and aug-cc-pVTZ. ${ }^{42}$ All interaction energies are counterpoise corrected with the procedure of Boys and Bernardi ${ }^{43,44}$ in order to account for the basis set superposition errors (BSSEs). Furthermore, we carry out Hartree-Fock calculations employing a 3-21G basis set.

In the following all energies are given for the example of an interacting dimer, a generalization to more complex systems is straightforward. The total intrinsic interaction energy for a dimer is defined as

$$
E_{I}\left(\mathbf{R}_{A}, \mathbf{R}_{B}\right)=E_{A B}\left(\mathbf{R}_{A}, \mathbf{R}_{B}\right)-E_{A}\left(\mathbf{R}_{A}\right)-E_{B}\left(\mathbf{R}_{B}\right),
$$

where $\mathbf{R}_{A}$ and $\mathbf{R}_{B}$ are the coordinates of the atoms of the monomers $A$ and $B$ in the complex, i.e., they are unrelaxed. $E_{A B}$ is the total energy of the dimer and $E_{A}$ and $E_{B}$ are the total energies of the monomers in the basis of the dimer. This requires that all monomer energies are counterpoise $(\mathrm{CP})$ corrected which is carried out with the procedure of Boys and Bernardi as already mentioned. ${ }^{43,44}$ The BSSEs lie between 13 and $29 \mathrm{~kJ} / \mathrm{mol}$ for Hartree-Fock, between 5 and $17 \mathrm{~kJ} / \mathrm{mol}$ for BP86, between 2 and $17 \mathrm{~kJ} / \mathrm{mol}$ for B3LYP, and between 28 and $46 \mathrm{~kJ} / \mathrm{mol}$ for MP2 per monomer depending on the configuration. In the following we omit for clarity the structure dependence $\left(\mathbf{R}_{A}, \mathbf{R}_{B}\right)$ and just use $E_{I}$ to indicate this interaction energy. A synonym that will be used for this energy is the calculated binding energy or just binding energy.

Energies that also incorporate the structural relaxation of the fragments are defined as the differences between total electronic energies of the dimer and of minimum structures,

$$
D_{e}=E_{A B}\left(\mathbf{R}_{A}, \mathbf{R}_{B}\right)-\left[E_{A}\left(\mathbf{R}_{A}^{\prime}\right)+E_{B}\left(\mathbf{R}_{B}^{\prime}\right)\right],
$$

where $\mathbf{R}_{A}^{\prime}$ and $\mathbf{R}_{B}^{\prime}$ are the coordinates of the atoms of the relaxed monomers $A$ and $B$. In the following this energy will 
TABLE I. Complexes that are used for the new SEN parametrization. The first molecule in the column "complex" denotes the proton donor, while the second is the acceptor. The table lists hydrogen bond distances in $\mathrm{pm}$ and the shared-electron number $\sigma_{H A}$ in $e$ and the binding energies in $\mathrm{kJ} / \mathrm{mol}$. All data are obtained from BP86/TZVP calculations.

\begin{tabular}{lccc}
\hline \hline Complex & $\Delta r(\mathrm{H} \cdots \mathrm{Cl})$ & $\sigma_{H A}$ & $E_{I}$ \\
\hline $\mathrm{HCl} \cdots \mathrm{HCl}$ & 247.2 & 0.0264 & -6.13 \\
$\mathrm{H}_{2} \mathrm{O} \cdots \mathrm{HCl}$ & 266.0 & 0.0137 & -2.49 \\
$\mathrm{H}_{2} \mathrm{~S} \cdots \mathrm{HCl}$ & 300.0 & 0.0059 & -0.62 \\
$\mathrm{PH}_{3} \cdots \mathrm{HCl}$ & 392.8 & 0.0004 & 0.59 \\
$\mathrm{NH}_{3} \cdots \mathrm{HCl}$ & 282.6 & 0.0089 & -0.95 \\
$\mathrm{PhOH} \cdots \mathrm{HCl}$ & 257.8 & 0.0186 & -3.76 \\
$\mathrm{PhSH} \cdots \mathrm{HCl}$ & 300.3 & 0.0060 & -0.13 \\
$\mathrm{~N}\left(\mathrm{CH}_{3}\right)_{3} \cdots \mathrm{HCl}$ & 272.9 & 0.0109 & -3.34 \\
$\mathrm{C}_{3} \mathrm{H}_{3} \mathrm{~N}_{2} \mathrm{CH} \cdots \mathrm{HCl}$ & 326.5 & 0.0021 & 0.48 \\
\hline \hline
\end{tabular}

also be called the adiabatic interaction energy or reaction energy. For comparison we can give the relative total energies of different complexes:

$$
\Delta E_{\mathrm{tot}}=E_{\mathrm{tot}}-E_{\mathrm{tot}}^{\mathrm{ref}}
$$

The index "ref" marks the configuration that serves as reference. All energies defined so far are given for $0 \mathrm{~K}$. Temperature effects are not included.

For static quantum chemical calculations on optimized structures it was found that the individual hydrogen bond energy can be estimated from the two-center shared-electron number $\sigma_{H A}(\mathrm{SEN}){ }^{45}$ The basic idea of the SEN method is to estimate the strength of a hydrogen bond by means of only one variable. This variable is the two-center shared-electron number $\sigma_{H A}$. It is roughly the number of electrons "that must be shared" by two atoms when in a population analysis elec-

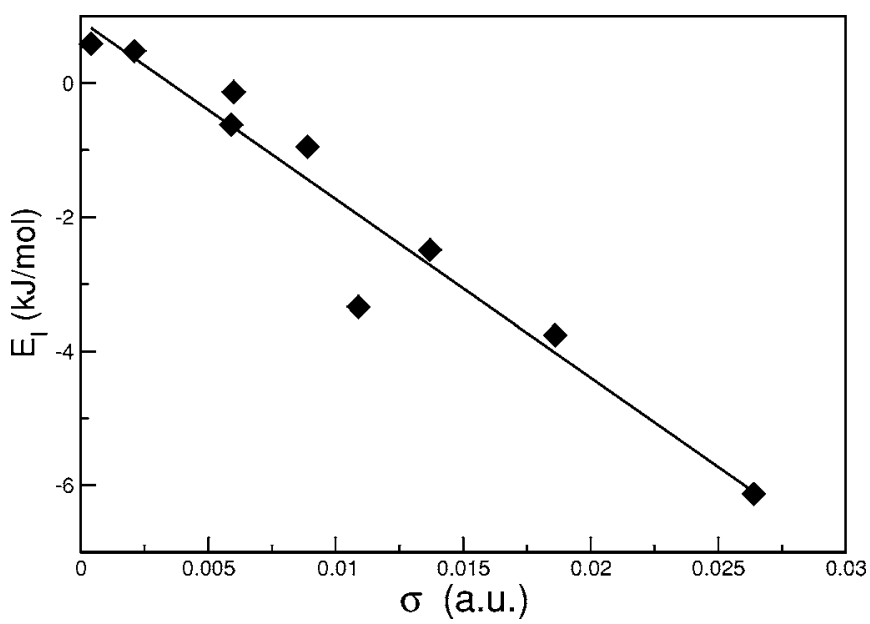

FIG. 1. Interaction energies plotted against the shared-electron number. The straight line gives the linear regression fitted against the calculated values given as diamonds.

trons are distributed to different atoms and the total amount of the distributed electrons is more than the available amount of electrons. ${ }^{4-49}$

Following Davidson ${ }^{46}$ one derives an occupation number $n_{a}$ of the atomic orbital $\varphi_{a}$ in a molecule which is described by the molecular orbital $\psi_{i}$ with the occupation $n_{i}(=1$ or 2$)$

$$
n_{a}=\sum_{i}\left|\left\langle\varphi_{a} \mid \psi_{i}\right\rangle\right|^{2} \cdot n_{i}
$$

The Pauli principle is obeyed: $2 \geqslant n_{a} \geqslant 0$ and the occupation number $N_{A}$ of an atom $A$ in the molecule reads

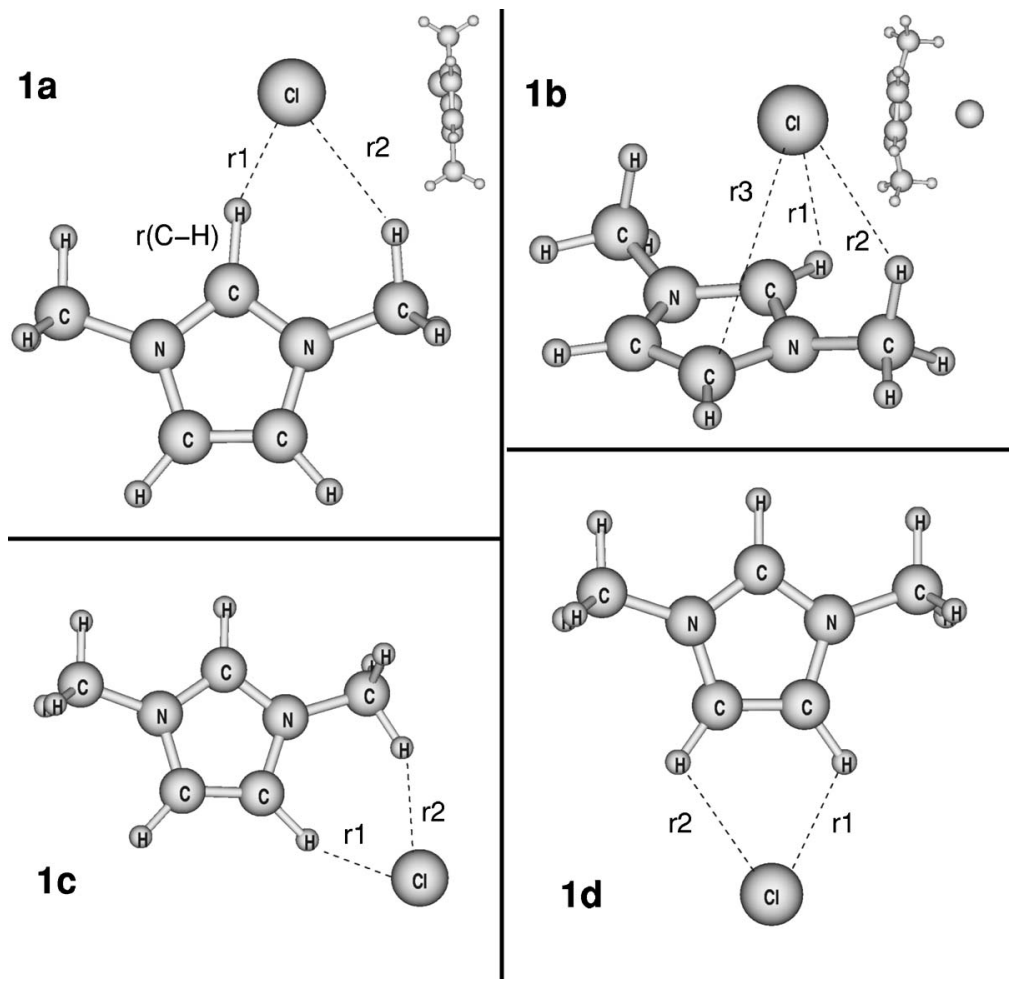

FIG. 2. Definition of distances and configuration of different monomer structures. Please note, distance $r 3$ in $1 \mathrm{~b}$ is not measured to the proton, but to the carbon atom. On the left for configurations $1 \mathrm{a}$ and $1 \mathrm{~b}$ the unit in the plane of the imidazolium is shown. 
TABLE II. Distances in pm, dipole moments $(d)$ in D, interaction energies $E_{l}$, and relative energies $\Delta E_{\text {tot }}$ with 1 a being the reference in $\mathrm{kJ} / \mathrm{mol}$ for different configurations of the $\left[\mathrm{C}_{1} \mathrm{C}_{1} i m\right] \mathrm{Cl}$ monomer unit. The $r(\mathrm{C}-\mathrm{H})$ distance is taken only at the most acidic proton. For configurations and definitions of distances, see Fig. 2. In configuration $1 \mathrm{~b}$ the distance $r 3$ is measured to a carbon atom. All data are obtained with the TZVP basis set, except HF which is calculated with the $3-21 \mathrm{G}$ basis set.

\begin{tabular}{|c|c|c|c|c|}
\hline & $\mathrm{HF}$ & BP86 & B3LYP & MP2 \\
\hline \multicolumn{5}{|c|}{ Complex 1a } \\
\hline$r 1$ & 213.5 & 190.7 & 196.7 & 194.2 \\
\hline$r 2$ & 257.6 & 252.8 & 258.5 & 250.7 \\
\hline$r(\mathrm{C}-\mathrm{H})$ & 108.4 & 115.4 & 112.7 & 112.2 \\
\hline$d$ & 14.3 & 11.7 & 12.4 & 12.7 \\
\hline$E_{I}$ & -433.4 & -449.9 & -404.6 & -372.2 \\
\hline$\Delta E_{\text {tot }}$ & 0.0 & 0.0 & 0.0 & 0.0 \\
\hline \multicolumn{5}{|c|}{ Complex 1b } \\
\hline$r 1$ & 247.5 & 259.9 & 260.6 & 279.1 \\
\hline$r 2$ & 293.6 & 283.5 & 287.4 & 274.0 \\
\hline$r 3$ & 424.5 & 383.8 & 390.3 & 355.4 \\
\hline$r(\mathrm{C}-\mathrm{H})$ & 105.0 & 108.1 & 107.2 & 107.2 \\
\hline$d$ & 11.3 & 7.2 & 8.4 & 8.5 \\
\hline$E_{I}$ & -362.6 & -427.5 & -409.3 & -384.7 \\
\hline$\Delta E_{\text {tot }}$ & 13.3 & -0.6 & 1.8 & -9.1 \\
\hline \multicolumn{5}{|c|}{ Complex 1c } \\
\hline$r 1$ & 227.8 & 205.4 & 212.7 & 208.9 \\
\hline$r 2$ & 239.0 & 227.7 & 231.9 & 227.4 \\
\hline$r(\mathrm{C}-\mathrm{H})$ & 106.4 & 108.4 & 107.6 & 107.5 \\
\hline$d$ & 18.4 & 15.4 & 16.2 & 16.9 \\
\hline$E_{I}$ & -347.4 & -378.6 & -369.1 & -339.0 \\
\hline$\Delta E_{\text {tot }}$ & 41.5 & 37.2 & 35.0 & 32.5 \\
\hline \multicolumn{5}{|c|}{ Complex $1 \mathrm{~d}$} \\
\hline$r 1$ & 258.1 & 237.5 & 246.6 & 246.3 \\
\hline$r 2$ & 258.3 & 254.0 & 254.5 & 251.9 \\
\hline$r(\mathrm{C}-\mathrm{H})$ & 106.4 & 108.4 & 107.6 & 107.5 \\
\hline$d$ & 18.9 & 15.7 & 16.7 & 17.7 \\
\hline$E_{I}$ & -315.9 & -347.6 & -337.8 & -318.1 \\
\hline$\Delta E_{\text {tot }}$ & 69.4 & 67.2 & 65.9 & 62.7 \\
\hline
\end{tabular}

$$
N_{A}=\sum_{a \in A} n_{a} .
$$

The number of electrons shared by two atoms within a molecule is defined as

$$
\sigma_{A B}=N_{A}+N_{B}-n_{A B} .
$$

Here $n_{A B}$ is the number of electrons contributed from atom $A$ and atom $B . N_{X}$ is calculated as

$$
N_{X}=\operatorname{tr} D P_{X},
$$

with $P_{X}$ being the projector onto the space of atomic orbitals of atom $X$, as indicated in Eq. (4). $D$ is the molecular oneparticle density operator.

The idea to relate SEN to a bond strength was examined by Ehrhardt and Ahlrichs for covalent bonds. ${ }^{49}$ In the original SEN approach ${ }^{45,50}$ for hydrogen bonds a linear relationship between the two-center SEN $\sigma_{H A}$ (obtained from population analysis ${ }^{46}$ ) and the energy of the hydrogen bond is assumed,

$$
f_{\text {tot }}^{\text {ori }}=m \sigma_{H A} \quad(\text { in } \mathrm{kJ} / \mathrm{mol}),
$$

where the index $H$ denotes the proton and $A$ denotes the hydrogen bond acceptor. Please note that this method has the feature to describe the directional nature of the hydrogen bond. Thereby the detection of hidden or unrecognized hydrogen bonds is possible. A useful application is the assignment of an interaction energy for a certain hydrogen bond when there are more than one of these present in a given hydrogen-bonded complex. We systematically investigated ${ }^{51}$ the shared-electron number method for a larger test set resulting in a fit of

$$
\left.f_{\text {tot }}=-354 \sigma_{H A}+1.61 \quad \text { (in } \mathrm{kJ} / \mathrm{mol}\right) \text {. }
$$

We found, furthermore, that large improvements can be expected if the SEN method is parametrized for the same acceptor atoms. ${ }^{51}$ Within this article we present such a fit for systems where molecules with a chloride atom are the acceptors. We optimize 25 complexes from which we can use nine, since the other complexes show multiple contacts.

From Table I and Fig. 1 it can be deduced that a strong hydrogen bond is given by a large SEN value, while a weak hydrogen bond is given by a SEN value close to zero. We obtained the following fit function:

$$
f_{\mathrm{Cl}}=-266 \sigma_{H A}+0.93 \quad \text { (in } \mathrm{kJ} / \mathrm{mol} \text { ). }
$$

As can be gathered from Eq. (10) we do not neglect the intercept. Physically this would mean that at zero sharedelectron number we would find a small amount of interaction. Thus the shared-electron number fit is more physical, if the intercept is fixed to zero. Nevertheless, in an extensive study we found a better agreement of the SEN fit with the hydrogen bond energy from the supermolecular approach when we add the intercept and therefore we decided to use an intercept. ${ }^{51}$ To choose the more reliable fit over the physical ansatz is not new and also done for potential energy surface fitting. If one calculates by means of static quantum chemical methods points on a potential energy surface and fits these points to an analytical function there are two different routes. Either one takes a physical function or one takes a function that best reproduces the calculations. If one is interested in getting the best numbers one certainly refrains from using the physical appropriate ansatz.

Atomic charges with multicenter corrections from the shared-electron population analysis are also given in some sections. ${ }^{46-49}$

We adapt the dihedral angles from the visualization program MOLDEN. ${ }^{52}$ Within this program the dihedral angle of an eclipsed conformation is defined as $0^{\circ}$. Negative values point in counterclockwise directions. Structures are also visualized with MOLDEN. ${ }^{52}$

\section{RESULTS}

\section{A. Monomers}

We denote the ion pair $\left[\mathrm{C}_{1} \mathrm{C}_{1}\right.$ im $]$ as monomer unit since we are interested in the behavior of this moiety and larger clusters formed by this monomer. Figure 2 depicts the four different monomer configurations we investigate. We opti- 
TABLE III. Distances $r$ in pm, dipole moments $(d)$ in $\mathrm{D}$, and interaction energies $E_{I}$ in $\mathrm{kJ} / \mathrm{mol}$ of different configurations of the $\left[\mathrm{C}_{1} \mathrm{C}_{1} i m\right] \mathrm{Cl}$ monomer units. The multicenter corrected SEN charges $(q)$ in a.u. on the chloride anion are shown. For pictures of configurations, see Fig. 2. All data are obtained with the TZVP basis set and the BP86 functional.

\begin{tabular}{ccccc}
\hline \hline No. & $1 \mathrm{a}$ & $1 \mathrm{~b}$ & $1 \mathrm{c}$ & $1 \mathrm{~d}$ \\
\hline$r 1$ & 190.7 & 259.9 & 205.4 & 246.6 \\
$r 2$ & 252.8 & 283.5 & 227.7 & 254.5 \\
$r(\mathrm{C}-\mathrm{H})$ & 115.4 & 108.1 & 108.4 & 108.4 \\
$d$ & 11.7 & 14.3 & 15.4 & 15.7 \\
$E_{I}$ & -449.9 & -427.5 & -378.6 & -347.6 \\
$q$ & -0.735 & -0.686 & -0.694 & -0.801 \\
\hline \hline
\end{tabular}

mize three structures, where the chloride lies in the "plane" of the imidazolium, see $1 \mathrm{a}, 1 \mathrm{c}$, and $1 \mathrm{~d}$. Configuration $1 \mathrm{~b}$ is an exception, since the chloride anion is positioned above the imidazolium ring. These configurations were previously detected as important motifs to construct the liquid or solid phase, see Refs. 18, 24, and 25 and reference therein.

We reinvestigate these monomer structures with different quantum chemical methods in order to gain insight in the quality of our choice of density functional and basis set combination. For the sake of practicability we chose the generalized gradient approximation density functional BP86 for our larger cluster studies. In Table II we list distances, dipole moments, and interaction energies as obtained for different methodologies with the TZVP basis set for each monomer configuration depicted in Fig. 2. We shall add here that the TZVP basis set quality is comparable to a basis set between the aug-cc-pVDZ and the aug-cc-pVTZ basis set. The TZVP basis set is thus superior to the standard 6-31 $\mathrm{G}^{\star \star}$ Pople basis set.

Intermolecular distances agree within $5 \mathrm{pm}$ for the monomers 1a and 1c comparing DFT and MP2. In 1d this distance depends more on the employed method. $1 \mathrm{~b}$ is probably the monomer where dispersion interactions play a more important role than in any other configuration. This is reflected in the large deviations between MP2 and DFT for all distances. Although Hartree-Fock can reproduce the intramolecular bonds in all monomers quite well as compared to the other methods, it largely fails to obtain accurate intermolecular distances. The intramolecular distances are all in agreement with each other at all configurations for different methods. Our distances compare well with previous investigations, ${ }^{24}$ where different functionals and a 6-31 $+\mathrm{G}^{\star \star}$ Pople basis set were employed. The optimized BP86/ TZVP distances agree within $3 \mathrm{pm}$ with the published BP86/6-31+G $\mathrm{G}^{\star \star}$ values. $^{24}$

The dipole moment shows the same trend for all methods. It has the value of 7-18 D, depending on the monomer unit and method. The following order is observed for all methods $1 \mathrm{~d}>1 \mathrm{c}>1 \mathrm{a}>1 \mathrm{~b}$.

For both the total and the binding energy the order 1a, $1 \mathrm{~b}<1 \mathrm{c}<1 \mathrm{~d}$ can be observed. Whether $1 \mathrm{a}$ or $1 \mathrm{~b}$ is the most stable configuration depends on the kind of energy and method chosen. For the binding energy $E_{I}$ we found 1 a to be more stable than $1 \mathrm{~b}$ for $\mathrm{HF}$ and $\mathrm{BP} 86$, while $1 \mathrm{~b}$ is more stable for B3LYP and MP2. In order to clarify the energetic behavior, we add more accurate calculations. Using a larger TZVPP basis set in combination with MP2 we find $E_{I}$ values of $-366.5 \mathrm{~kJ} / \mathrm{mol}$ for $1 \mathrm{a}$ and $-376.6 \mathrm{~kJ} / \mathrm{mol}$ for $1 \mathrm{~b}$. This confirms the trend observed before. According to MP2 the total energy shows the same trend. This is in agreement with previous calculations. ${ }^{24,25}$ Del Pópolo et al. ${ }^{25}$ gave an energy difference between $1 \mathrm{~b}$ and $1 \mathrm{a}$ of $-13.5 \mathrm{~kJ} / \mathrm{mol}$, whereas Bühl et al. ${ }^{24}$ listed a difference of $-5.0 \mathrm{~kJ} / \mathrm{mol}$ for MP2 (we assume they compare total energies). The gradient corrected functional BP86 yields also a slightly more stable complex $1 \mathrm{~b}$ in contrast to Bühl et al., who find $1 \mathrm{~b}$ to be $2 \mathrm{~kJ} / \mathrm{mol}$ less stable than 1a for BP86/6-31+ $\mathrm{G}^{\star \star} \cdot{ }^{24}$ This can only be attributed to the different basis sets. Surprisingly, the total energies obtained by B3LYP give an opposite trend to MP2 in our calculation as well as in the calculation of Ref. 24. A final answer to this stability question can only be expected from an even more accurate electronic structure method such as coupled cluster combined with a complete basis set.

For the larger clusters we chose in the following configuration 1a since the geometrical correspondence with the other electronic structure models is closer than for structure 1b. It is likely that the discrepancies in the energetic order stem from the wrong description of monomer $1 b$ rather than
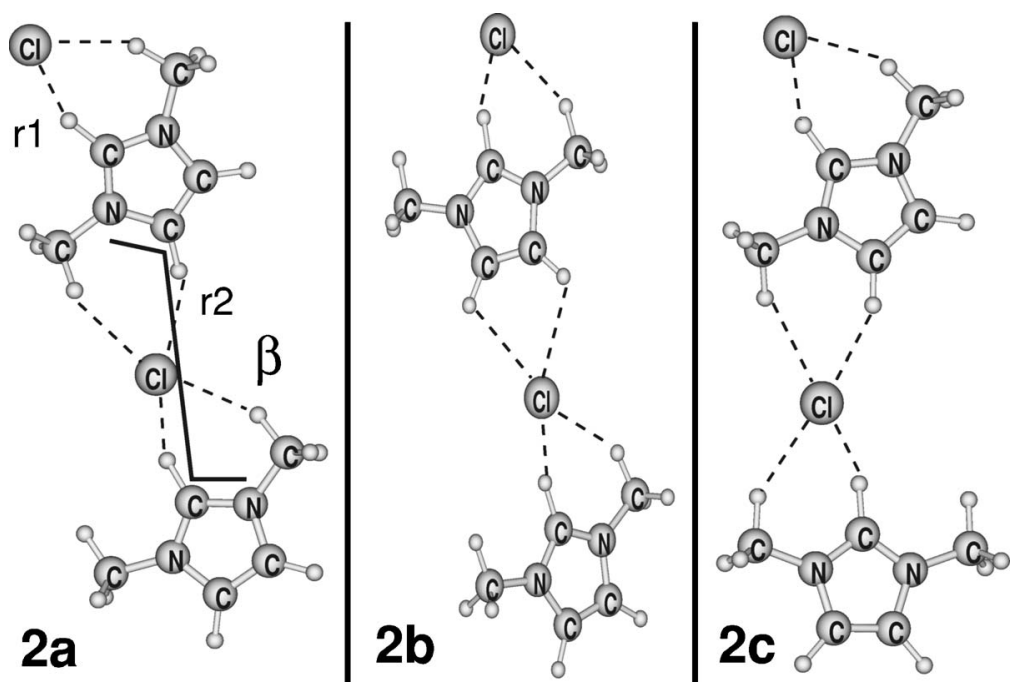

FIG. 3. Definition of distances and configurations of different dimer structures. $\beta$ indicates the NCCN dihedral angle between the planes of the two $\left[\mathrm{C}_{1} \mathrm{C}_{1}\right.$ im $]$ units. 


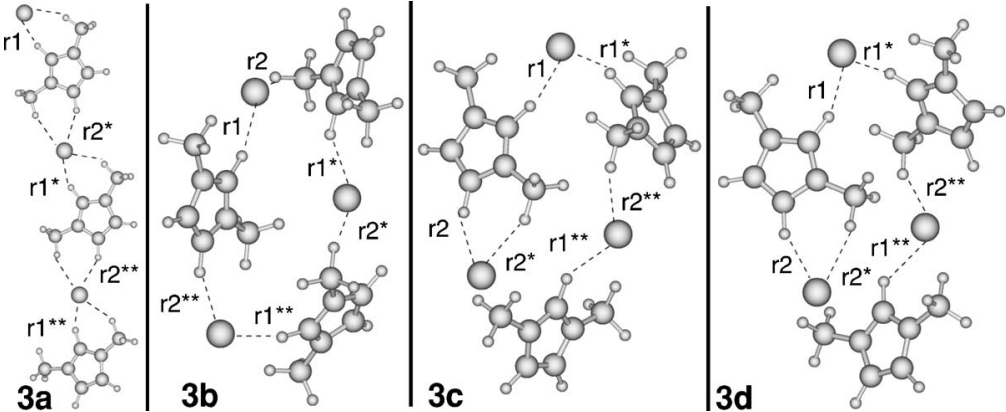

FIG. 4. Definition of distances and configurations of different trimer structures. Please note that the common element for $r 2$ is not structural, i.e., at the same atom, but it is for all the second shortest distance. of monomer $1 \mathrm{a}$, because the monomer $1 \mathrm{~b}$ is a typical example for large dispersion interactions and such complexes are known to be described rather poor by standard density functional theory (with gradient corrected functionals). ${ }^{53-56}$

We turn now to the comparison of the different monomer configurations and concentrate on BP86 values.

Depending on the location of the chloride anion the distance $r 1$ between the proton donated by the imidazolium cation and the chloride anion changes. Upon coordination the $\mathrm{C}-\mathrm{H}$ distance is elongated by about $7 \mathrm{pm}$, compare third line of Table III configuration $1 \mathrm{a}(r(\mathrm{C}-\mathrm{H})=115.4 \mathrm{pm})$ with the other configurations $(r(\mathrm{C}-\mathrm{H})=108 \mathrm{pm})$. This is also the most acidic proton. The dipole moments are in the range of 11-16 D. Although this is not new, it is still surprising when compared to usual molecular liquid units such as dimethyl sulfoxide (DMSO) where the isolated gas phase value of the DMSO dipole moment is about $3.96 \mathrm{D} .^{57}$ The interaction energies are very large due to electrostatic interaction formally of two separated charges. We also note that the bond elongation $r(\mathrm{C}-\mathrm{H})$, the dipole moment, and the calculated binding energy $E_{I}$ correspond with each other. Conformer $1 \mathrm{~d}$ exhibits the largest negative value for the charge. For obvious reasons the charge on the chloride atom is always negative.

\section{B. Dimer clusters}

The dimer configurations investigated are shown in Fig. 3. The dimers are always connected in such a way that all monomer structures lie in a plane. The monomer units al-

TABLE IV. Distances in pm, dipole moments $(d)$ in $\mathrm{D}$, and interaction energies $E_{I}$ in $\mathrm{kJ} / \mathrm{mol}$ for different configurations of the $\left[\mathrm{C}_{1} \mathrm{C}_{1}\right.$ im $] \mathrm{Cl}$ dimer units. The first energy block considers the energies between all ions, whereas the second block considers dissociation into monomer pairs. For pictures of configurations, see Fig. 3. All data are obtained with the TZVP basis set and the BP86 functional.

\begin{tabular}{lrrr}
\hline \hline Complex & $2 \mathrm{a}$ & $2 \mathrm{~b}$ & \multicolumn{2}{c}{$2 \mathrm{c}$} \\
\hline$r 1$ & 201.1 & 197.4 & 201.4 \\
$r 2$ & 247.8 & 285.8 & 247.2 \\
$d$ & 27.4 & 26.8 & 27.8 \\
& \multicolumn{2}{c}{ Ions } \\
$E_{I}$ & -866.9 & -858.6 & -866.8 \\
& \multicolumn{2}{c}{ Monomer pair } \\
$E_{I}$ & -49.4 & -36.5 & -49.7 \\
$D_{e}$ & -44.3 & -34.3 & -44.1 \\
\hline \hline
\end{tabular}

ways consist of the lowest energy monomer unit 1a. An extension of this study will of course consider different dimer configurations where other monomer units are also incorporated within the clusters. We find similar geometries for dimers $2 \mathrm{a}$ and $2 \mathrm{c}$. The only difference between them is that the free methyl group in $2 \mathrm{a}$ is further away from the first imidazolium cation than in $2 \mathrm{c}$. Obviously this is not that important. Much more important is the connectivity between the monomer units. In $2 \mathrm{~b}$ the two monomers are interconnected as in structure $1 \mathrm{~d}$ of the monomer unit. This costs approximately $10 \mathrm{~kJ} / \mathrm{mol}$ of binding energy, see Table IV. It is readily apparent from the second energy block that the monomer units must hold a very optimal geometry, since there is almost no difference between the binding energy $E_{I}$ and the reaction energy $D_{e}$. The slightly larger difference between $E_{I}$ and $D_{e}$ for $2 \mathrm{a}$ and $2 \mathrm{c}$ as compared to $2 \mathrm{~b}$ correlates with their shorter distance $r 2$, i.e., the monomer units are closer together and are thus more distorted. As we now formally have arrived at four separated charges, the total interaction energies $E_{I}$ are much larger than in the case of the monomer unit. If we consider the average energy per unit we calculate $-433.5 \mathrm{~kJ} / \mathrm{mol}$ for $2 \mathrm{a},-429.3 \mathrm{~kJ} / \mathrm{mol}$ for $2 \mathrm{~b}$, and $-433.4 \mathrm{~kJ} / \mathrm{mol}$ for $2 \mathrm{c}$. These energies are all by $20 \mathrm{~kJ} / \mathrm{mol}$ weaker than the energy of monomer 1a. This means that in the gas phase these dimers would dissociate into monomers from a pure energetical point of view neglecting kinetics.

\section{Trimer clusters}

In Fig. 4 different configurations of trimers are shown. The first trimer is the one that logically forms from building up monomer 1a and dimer $2 \mathrm{a}$. In the subsequent section we will form a chain of up to nine monomer units from this configuration. Trimer $3 \mathrm{~b}$ is a cyclic structure, so is $3 \mathrm{c}$ and $3 \mathrm{~d}$. Whereas the connectivity of $3 \mathrm{~b}$ is always of the pattern that connects one chloride with the acidic $\mathrm{C}-\mathrm{H}$ position of the imidazolium cation, in $3 \mathrm{c}$ one chloride anion is shared between this position of two imidazolium cations, see $r 1$ and $r 1^{\star} .3 \mathrm{~d}$ is originally built from monomer unit $1 \mathrm{~b}$, but after optimization it rearranges to another cyclic configuration.

In Table $\mathrm{V}$ we show the characteristic parameters.

The distance $r 1(=203.7 \mathrm{pm})$ is elongated in $3 \mathrm{a}$ as compared to $r 1(=190.7 \mathrm{pm})$ in $1 \mathrm{a}$ and $r 1(=201.1 \mathrm{pm})$ in $2 \mathrm{a}$. Furthermore the middle distance $r 1^{\star}$ is elongated by more than $10-20$ pm. $r 1^{\star \star}$ is again of the same length as $r 1$. We note that this is the first cooperative effect observed in this article and we will further discuss these cooperative effects in the subsequent section. For the cyclic structures these dis- 
TABLE V. Distances in pm for different configurations of the $\left[\mathrm{C}_{1} \mathrm{C}_{1}\right.$ im $] \mathrm{Cl}$ trimers. The first block considers the distance $r 1$, as defined in Fig. 4 . The distances for $3 b$ and $3 c$ are not precisely defined. Therefore we chose for $r 1$ the shortest hydrogen bond and for $r 2$ the shortest bond to the next imidazolium. $r(\mathrm{C}-\mathrm{H})$ is the distance of the most acidic proton in the imidazolium, see Fig. 4 for explanations. We also give charges in a.u. on the chloride anion corresponding to distance $r 1 . \varnothing$ gives the averaged values. All data are obtained with the TZVP basis set and the BP86 functional.

\begin{tabular}{|c|c|c|c|c|}
\hline & $r$ & $r \star$ & $r \star \star$ & $\varnothing_{r}$ \\
\hline \multicolumn{5}{|c|}{$r 1$} \\
\hline $3 a$ & 203.7 & 214.9 & 204.6 & 207.7 \\
\hline $3 b$ & 226.3 & 233.3 & 221.3 & 227.0 \\
\hline $3 c$ & 226.1 & 227.0 & 233.5 & 228.9 \\
\hline $3 d$ & 232.5 & 224.5 & 237.9 & 231.6 \\
\hline \multicolumn{5}{|c|}{$r 2$} \\
\hline $3 a$ & $\cdots$ & 242.6 & 241.7 & 242.2 \\
\hline $3 b$ & 267.9 & 245.0 & 238.0 & 250.3 \\
\hline $3 c$ & 235.0 & 244.2 & 245.4 & 241.5 \\
\hline $3 d$ & 231.7 & 256.9 & 242.5 & 243.7 \\
\hline \multicolumn{5}{|c|}{$r(\mathrm{C}-\mathrm{H})$} \\
\hline $3 a$ & 113.0 & 111.2 & 112.3 & 112.2 \\
\hline $3 b$ & 110.3 & 109.6 & 110.2 & 110.0 \\
\hline $3 c$ & 110.3 & 110.0 & 109.7 & 110.0 \\
\hline $3 d$ & 110.0 & 110.1 & 109.4 & 109.8 \\
\hline \multicolumn{5}{|c|}{$q$} \\
\hline $3 a$ & -0.785 & -0.801 & -0.780 & -0.789 \\
\hline $3 b$ & -0.817 & -0.839 & -0.796 & -0.817 \\
\hline $3 c$ & -0.796 & -0.833 & -0.839 & -0.823 \\
\hline $3 d$ & -0.798 & -0.816 & -0.840 & -0.818 \\
\hline
\end{tabular}

tances fall in the range of approximately 230 pm, i.e., they are much larger than in the chains, see also averaged values given in the fourth entry of Table $\mathrm{V}$. The distance $r 2$ is then again comparable for all conformers. Please note that the common element for $r 2$ is not structural, i.e., at the same atom, but it is for all the second shortest distance. In the cyclic configurations this means that the $r 2$ distances are now only $10 \mathrm{pm}$ longer than the $r 1$ distances. This small difference still marks the $r 1$ positions as most important attack site. However, the difference is significantly reduced compared to the chains. This is also expressed in the mean

TABLE VI. Dipole moments $(d)$ in D, and interaction energies $E_{I}$ in $\mathrm{kJ} / \mathrm{mol}$ for different configurations of the $\left[\mathrm{C}_{1} \mathrm{C}_{1} i m\right] \mathrm{Cl}$ trimers. The first energy block considers the binding energies between all ions, whereas the second block considers dissociation into monomer pairs. For pictures of configurations, see Fig. 4. All data are obtained with the TZVP basis set and the BP86 functional.

\begin{tabular}{lrcrr}
\hline \hline & $3 \mathrm{a}$ & $3 \mathrm{~b}$ & $3 \mathrm{c}$ & $3 \mathrm{~d}$ \\
\hline$d$ & 44.5 & 7.0 & 3.8 & 4.4 \\
& & & & \\
& & Ions & & -1380.3 \\
$E_{I}$ & -1329.9 & -1385.8 & -1380.3 & -460.1 \\
$E_{I} / 3$ & -443.3 & -461.9 & -460.1 & \\
& & & & -258.0 \\
$E_{I}$ & -117.0 & -207.0 & -256.9 & -80.9 \\
$E_{I} / 3$ & -37.4 & -64.4 & -81.3 & \\
\hline \hline
\end{tabular}
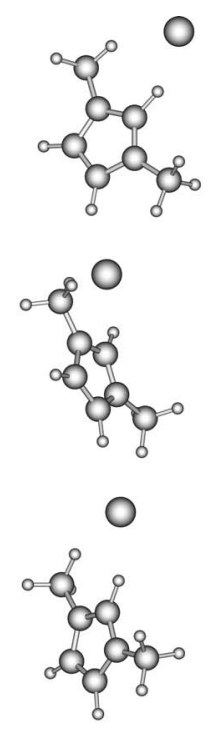

0
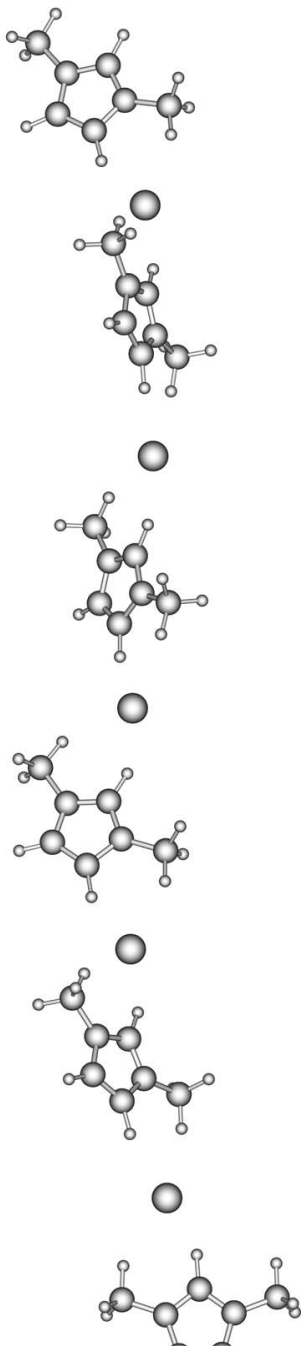

FIG. 5. Configuration of the investigated nonamer 9a. All smaller clusters are started from the same connectivity pattern.

values $\varnothing$ r. A similar effect was observed in the 1-ethylmethylimidazolium- $n$-chloride study of Wang et al. ${ }^{18}$ Whereas their single coordinated pair gives an analogous $r 1$ distance of $198.8 \mathrm{pm}$, the distance is elongated to $222.4 \mathrm{pm}$ 
TABLE VII. Distances in pm. For definition of distances, see Figs. 2 and 3. The symbol " $\varnothing$ " indicates an average over distances in one particular cluster. The difference to the value of the monomer $\Delta$ is also given. All data are obtained with the TZVP basis set and the BP86 functional.

\begin{tabular}{lcrrrrrrrrr}
\hline \hline No. & $1 \mathrm{a}$ & \multicolumn{1}{c}{$2 \mathrm{a}$} & \multicolumn{1}{c}{$3 \mathrm{a}$} & \multicolumn{1}{c}{$4 \mathrm{a}$} & $5 \mathrm{a}$ & \multicolumn{1}{c}{$6 \mathrm{a}$} & $7 \mathrm{a}$ & $8 \mathrm{a}$ & $9 \mathrm{a}$ & $\infty$ \\
\hline$r 1$ & 190.7 & 201.4 & 203.6 & 204.2 & 204.5 & 204.7 & 204.8 & 205.1 & 205.4 & 207.7 \\
$\Delta r 1$ & $\ldots$ & 10.7 & 12.9 & 13.5 & 13.8 & 14.0 & 14.1 & 14.4 & 14.7 & 15.7 \\
$\varnothing r 1$ & 190.7 & 201.1 & 207.5 & 210.1 & 212.1 & 213.8 & 215.2 & 216.2 & 216.9 & 218.6 \\
$\Delta \varnothing r 1$ & $\ldots$ & 10.4 & 16.8 & 19.4 & 21.4 & 23.1 & 24.5 & 25.5 & 26.2 & 30.1 \\
$r 2$ & $\ldots$ & 247.2 & 241.9 & 244.7 & 244.1 & 243.7 & 243.6 & 243.3 & 243.0 & 242.2 \\
$\varnothing(r 2)$ & $\ldots$ & 247.2 & 241.5 & 242.3 & 241.2 & 239.5 & 238.8 & 238.0 & 237.7 & 235.6 \\
$r(\mathrm{C}-\mathrm{H})$ & 115.4 & 113.3 & 113.0 & 112.9 & 112.8 & 111.4 & 112.8 & 112.8 & 112.7 & 112.0 \\
$\Delta r(\mathrm{C}-\mathrm{H})$ & $\ldots$ & -2.1 & -2.4 & -2.5 & -2.6 & -2.6 & -2.6 & -2.6 & -2.7 & -2.8 \\
$\varnothing(r(\mathrm{C}-\mathrm{H}))$ & 115.4 & 113.1 & 112.2 & 111.8 & 111.6 & 111.4 & 111.3 & 111.1 & 111.1 & 110.6 \\
$\Delta \varnothing(r(\mathrm{C}-\mathrm{H}))$ & $\cdots$ & -2.3 & -3.2 & -3.6 & -4.0 & -4.1 & -4.1 & -4.3 & -4.3 & -4.9 \\
\hline \hline
\end{tabular}

when a second chloride is attached at a second proton of the imidazolium unit, see Figs. 3 and 4 of Ref. ${ }^{18}$.

Inverse effects of the behavior in $r 1$ can be found in the $r(\mathrm{C}-\mathrm{H})$ distance. Namely, $r(\mathrm{C}-\mathrm{H})^{\star}$ is shorter than $r(\mathrm{C}-\mathrm{H})$ or $r(\mathrm{C}-\mathrm{H})^{\star \star}$. In the cyclic structures $3 \mathrm{~b}, 3 \mathrm{c}$, and $3 \mathrm{~d}$ these bonds are almost unaffected by the chloride coordination. Not surprisingly, the charges on the chloride are correlated to the $r 1$ series. The larger the distance, the larger the charges. On average the charges in the cyclic trimers are larger than the ones in the chain.

The dipole for the chain is very large. This is not surprising considering the fact that the dipole moments of all monomer units point into a similar direction. As opposed to this the dipole moments of all three cyclic structures $3 b, 3 c$, and $3 \mathrm{~d}$ are much smaller, i.e., they are closer to the dipole moments of molecular liquid units. From this we may ask whether these motifs could play a role in the liquid phase. Measurements of polarity by Wakai et al. ${ }^{58}$ indicated an only moderate polarity $(15.2 \leqslant \epsilon \leqslant 8.8)$, as opposed to Reichardt $(\epsilon \approx 32.5) .{ }^{59}$ Even the higher polarity is comparable to short chain alcohols, which exhibit a much lower dipole moment than our ionic liquid monomer units. Thus, we might infer that such cyclic structures do play a role in the liquid phase, since in the crystal structures of imidazolium halides the cations and anions are arranged in extended networks. ${ }^{60} \mathrm{We}$ mentioned before that the distance $r 1$ displays cooperative

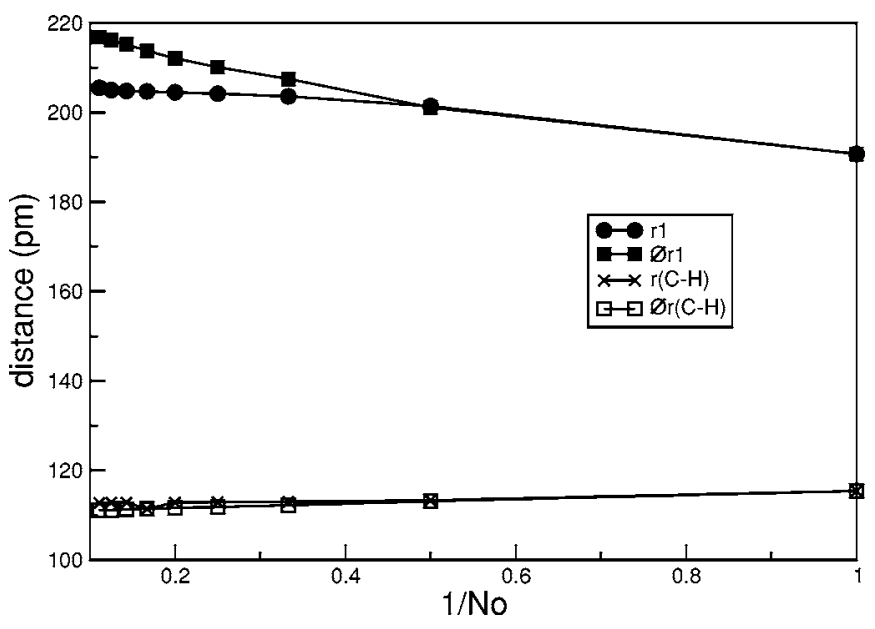

FIG. 6. Distances depicted against the inverse number of clusters. effects. Additional cooperative effects can be found in the energies. Both the $E_{I}$ of the dissociation into ions and of the dissociation into pairs are much larger for the cyclic structures than for the chains. If we consider for the dissociation into ions the contribution per monomer unit we realize that $3 \mathrm{~b}, 3 \mathrm{c}$, and $3 \mathrm{~d}$ are stronger bound than 1a. Additionally, the ring trimers are more stable than the dimers and the chain trimer $3 \mathrm{a}$. The stability of the monomer $1 \mathrm{a}$ is achieved for a chain structure at the tetramer configuration, where we find
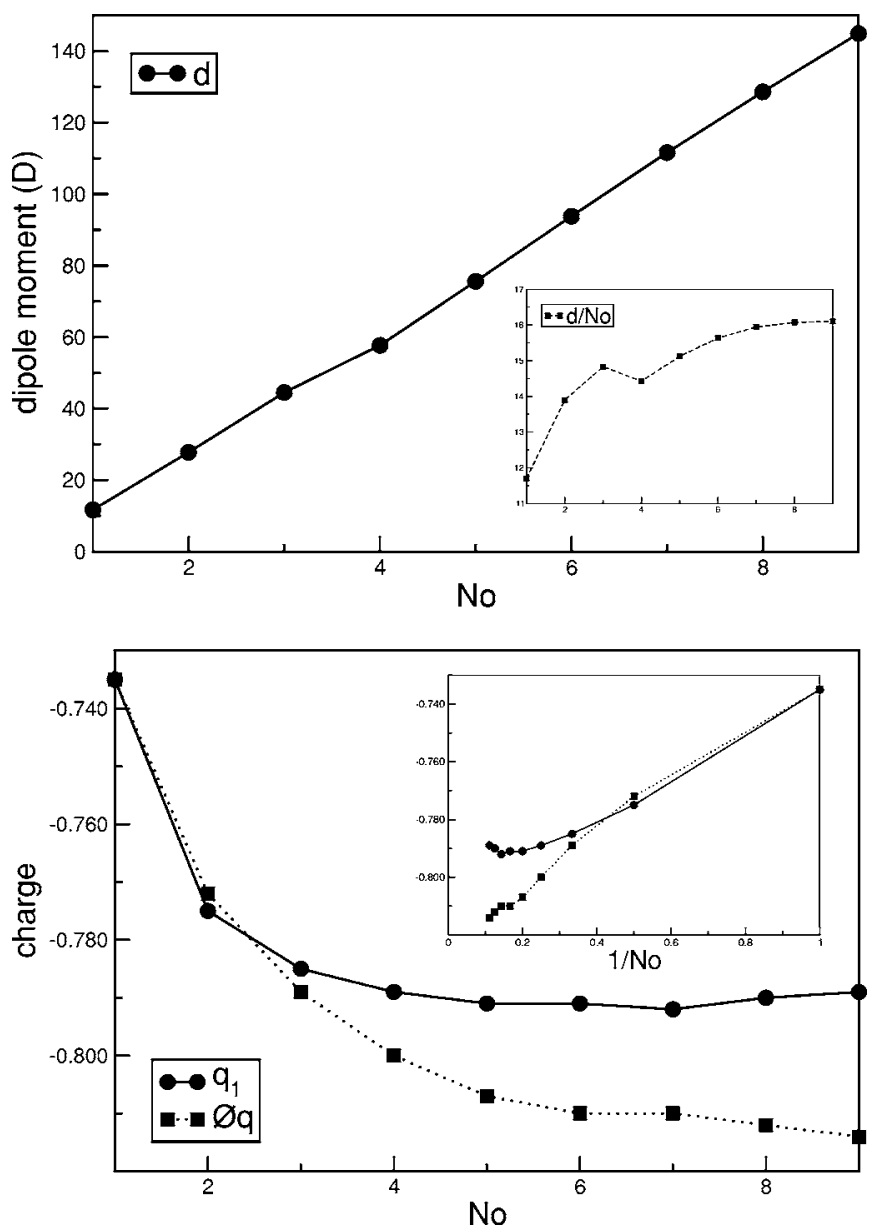

FIG. 7. Above: dipole moments plotted against the number of units. The inlet shows the dipole moment divided by the number of units against the number of units. Below: the charge on the chloride atom with the number of units. Inlet: charges plotted against the inverse of the number of monomer units. 
TABLE VIII. Dipole moments $(d)$ in D. Charges $(q)$ in a.u. on the chloride anion are also listed. Whereas the index 1 denotes the charge on the first chloride anion, the symbol " $\varnothing$ " indicates the charge averaged over all chloride anions. All data are obtained with the TZVP basis set and the BP86 functional.

\begin{tabular}{lccccccccccc}
\hline \hline No. & $1 \mathrm{a}$ & $2 \mathrm{a}$ & $3 \mathrm{a}$ & $4 \mathrm{a}$ & $5 \mathrm{a}$ & $6 \mathrm{a}$ & $7 \mathrm{a}$ & $8 \mathrm{a}$ & $9 \mathrm{a}$ & $\infty$ \\
\hline$d$ & 11.7 & 27.4 & 44.5 & 57.7 & 75.6 & 93.8 & 111.6 & 128.6 & 144.9 & $\ldots$ \\
$d /$ No. & 11.7 & 13.9 & 14.8 & 14.4 & 15.1 & 15.6 & 15.9 & 16.1 & 16.1 & 16.3 \\
$q_{1}$ & -0.735 & -0.775 & -0.785 & -0.789 & -0.791 & -0.791 & -0.792 & -0.790 & -0.789 & -0.802 \\
$\varnothing q_{1}$ & -0.735 & -0.772 & -0.789 & -0.800 & -0.807 & -0.810 & -0.810 & -0.812 & -0.814 & -0.823 \\
\hline \hline
\end{tabular}

an interaction energy of $E_{I}=1800.2 \mathrm{~kJ} / \mathrm{mol} \quad\left(E_{I} / 4\right.$ $=450.1 \mathrm{~kJ} / \mathrm{mol})$. Large cooperative effects are demonstrated by the interaction energies between monomer units, see second energy block of Table VI. $E_{I}$ between the monomer units in the ring configurations exceeds the corresponding energy of the trimer chain by more than $100 \mathrm{~kJ} / \mathrm{mol}$. If we refer to the energy per contact $E_{I} / 3$ all timers are more stable than the dimers. The ring trimers hold monomer units that resemble 1c which is less stable than 1a, see previous sections.

\section{Cooperative effects}

We construct from the monomer $1 \mathrm{a}$ and the dimer $2 \mathrm{a}$ higher aggregates to investigate cooperative effects. Figure 5 shows the highest cluster we consider, namely, the nonamer structure 9a. We refrain from constructing larger ring systems. One reason for this is that the smallest possible size of the cluster to start with is a trimer; this would neglect considerations for the monomer and the dimer. But a comparison to smaller clusters such as the monomer and dimer is especially interesting since many theoretical studies are carried out at small isolated systems, mostly one pair. A second reason is that it is often observed that only a few ring sizes are stable in liquids. This does not allow us to plot quantities with an increasing size of unit without having singularities in our curves. Ring structures exhibit many more different effects (e.g., angle dependency) than pure solvation effects that can be captured with chain structures. Thus ring systems cannot be studied systematically if one aims at investigating properties with an increasing cluster size. Furthermore, before we compared the polarity of this ionic liquid with small alcohol systems. For liquid methanol both experimental and theoretical data suggest that the dominant structure is a series of chains rather than a topologically more complex threedimensional hydrogen-bonded arrangement. ${ }^{61}$

From the possible chains we chose the one built up by 1a for the reasons given in the previous chapter. Furthermore
Del Pópolo et al. ${ }^{25}$ and Bühl et al. ${ }^{24}$ found that this motif is more likely to play a role in the liquid phase than $1 \mathrm{~b}$. This is confirmed by neutron diffraction experiments. ${ }^{62}$

Although all clusters are started from the same connectivity pattern, upon optimization they sometimes arrange differently in the orientation of the monomer units, i.e., sometimes all monomer units are more or less within one plane and sometimes not. We will come back to this point in a subsequent section.

\section{Geometry}

We measure some characteristic distances in the clusters. The first distance listed in Table VII is the $r 1$ distance. This distance is analogous to a hydrogen bond distance in $\mathrm{H}_{2} \mathrm{O} \cdots \mathrm{H}_{2} \mathrm{O}$ and is defined in several figures shown before. Upon increasing chain length the $r 1$ distance increases. This is also the case for the average $\varnothing(r 1)$ distance (difference to the previous structure). We observe that this distance displays cooperative effects. The bond elongations $\Delta r 1$ and $\Delta \varnothing r 1$ are given in the next line. Whereas the $r 1$ elongation is half the size of the increase in a water chain, see Ref. 63, the increase of the average $\varnothing r 1$ distance is twice as large as compared to the water chain. The $r 2$ distance decreases, although there is one very short distance for the $3 \mathrm{a}$ trimer as compared to any other cluster. This behavior averages out when the mean value of the $r 2$ distance is calculated.

Figure 6 shows the distances plotted against the inverse of the number of monomer units in the clusters. Such a plot allows a linear regression to be fitted to the data and to derive the value at cluster size $\infty$ from the intercept. For the $r 1$ and $\varnothing r 1$ we get $\infty$ units values of 207.8 and 218.5 pm, respectively. In Ref. 25 the radial distribution functions are calculated. The particular function for $\mathrm{H} \cdots \mathrm{Cl}$ shows a first peak around $220 \mathrm{pm}$ which is in agreement with our $\infty$ $-\varnothing r 1$ distance of $218.9 \mathrm{pm}$. It is also in agreement with the cyclic structure with the $\varnothing r 1$ distance of 227.0 (228.9) pm

TABLE IX. The N-C $\cdots \mathrm{C}-\mathrm{N}$ dihedral angle $\beta$, see Fig. 3 and configuration $2 \mathrm{a}$ for explanation. All data are obtained with the TZVP basis set and the BP86 functional.

\begin{tabular}{|c|c|c|c|c|c|c|c|c|}
\hline No & 1 & 2 & 3 & 4 & 5 & 6 & 7 & 8 \\
\hline $2 \mathrm{a}$ & -167.6 & $\ldots$ & $\cdots$ & $\cdots$ & $\cdots$ & $\ldots$ & $\cdots$ & $\cdots$ \\
\hline $3 a$ & -174.0 & -165.7 & $\ldots$ & $\cdots$ & $\ldots$ & $\ldots$ & $\ldots$ & $\ldots$ \\
\hline $4 a$ & -99.0 & 111.7 & -156.8 & $\cdots$ & $\cdots$ & $\cdots$ & $\cdots$ & $\cdots$ \\
\hline $5 a$ & -98.7 & 92.8 & -93.2 & 139.5 & $\cdots$ & $\ldots$ & $\ldots$ & $\cdots$ \\
\hline $6 a$ & -80.8 & 106.6 & 126.4 & -77.1 & 129.8 & $\ldots$ & $\ldots$ & $\ldots$ \\
\hline $7 \mathrm{a}$ & -82.8 & 111.1 & 132.1 & -68.4 & 102.0 & -178.7 & $\cdots$ & $\cdots$ \\
\hline $8 \mathrm{a}$ & -81.1 & 110.3 & 125.3 & -54.4 & 95.5 & 151.4 & -144.2 & $\cdots$ \\
\hline $9 a$ & 97.5 & -68.5 & 138.6 & 101.2 & -57.6 & 128.4 & 130.1 & -137.5 \\
\hline
\end{tabular}


TABLE X. SEN hydrogen bond energies $f_{\mathrm{Cl}}$. All energies are given in $\mathrm{kJ} / \mathrm{mol}$. Whereas the index 1 denotes the hydrogen bond to the proton defined by the distance $r(\mathrm{C}-\mathrm{H})$, see Fig. 5 to the first chloride, the index " $\varnothing$ " means average hydrogen bond per chloride to all protons. All data are obtained with the TZVP basis set and the BP86 functional.

\begin{tabular}{lccccccccrr}
\hline \hline No. & $1 \mathrm{a}$ & $2 \mathrm{a}$ & $3 \mathrm{a}$ & $4 \mathrm{a}$ & $5 \mathrm{a}$ & $6 \mathrm{a}$ & $7 \mathrm{a}$ & $8 \mathrm{a}$ & $9 \mathrm{a}$ & $\infty$ \\
\hline$f_{\mathrm{Cl}, 1}$ & -43.2 & -32.8 & -30.8 & -30.1 & -29.9 & -29.7 & -29.7 & -29.4 & -29.2 & -26.8 \\
$f_{\mathrm{Cl}, \varnothing}$ & -25.3 & -14.5 & -12.2 & -11.0 & -10.5 & -10.2 & -10.0 & -9.8 & -9.7 & -7.1 \\
\hline \hline
\end{tabular}

for $3 \mathrm{~b}(3 \mathrm{c})$. The $r 2$ distances decrease with an increasing number of $\left[\mathrm{C}_{1} \mathrm{C}_{1} \mathrm{im}\right] \mathrm{Cl}$ units. The extrapolated values for $r 2$ and $\varnothing \mathrm{r} 2$ are 242.2 and $235.6 \mathrm{pm}$. Distances of chloride to the hydrogens of $\left[\mathrm{C}_{1} \mathrm{C}_{1} i \mathrm{im}\right]$ in neutron diffraction measurements were found by Hardacre et al. to be in the range of $260-290 \mathrm{pm}$ at $425 \mathrm{~K}^{62}$

Turning now to the $\mathrm{C}-\mathrm{H}$ distance, we observe a bond contraction with an increasing chain length. This contraction is much larger than the contraction of the $\mathrm{C}-\mathrm{H}$ distance in a $\mathrm{C}-\mathrm{H}$...O hydrogen bond of $\mathrm{HCHO}$ chains, see Ref. 63. Whereas no cooperative effect is observed for this kind of bond in $\mathrm{HCHO}$, a smaller but noticeable cooperative effect can be seen in the $r(\mathrm{C}-\mathrm{H})$ and the $\varnothing(r(\mathrm{C}-\mathrm{H}))$ distance in our ionic liquid model. Both cooperative effects in the $r 1$ and $r(\mathrm{C}-\mathrm{H})$ bonds, i.e., the decrease and increase in the bonds, are depicted in Fig. 6.

\section{Dipole moments and charges}

We observe from Fig. 7 and Table VIII that the dipole moment increases almost linearly with the number of monomer units. The dipole moment per monomer unit also increases, but not linearly and an extrapolation to $\infty$ units gives a dipole moment of $16.3 \mathrm{D}$. Around four to five monomer units there seems to be a transition, which manifests also in $\Delta \varnothing r 1$, see Fig. 6. Upon examination of the structure we find different orientations of the monomer units, as mentioned at the beginning of this section. To understand these transitions we consider the dihedral angles. In Table IX the dihedral angles $\beta$ are given. See Fig. 3 for explanation of $\beta$. We see that up to the trimer the units lie almost in 4 a plane. From the tetramer $4 \mathrm{a}$ on the units are twisted. Whereas the pentamer and the tetramer are always twisted in the opposite direction, the higher $n$-mers present configurations where the units are twisted twice in the same direction, see Table IX structure $7 \mathrm{a}$ $\beta-2$ and $\beta-3$, structure $8 \mathrm{a} \beta 2$ and $\beta-3$, and structure 9a $\beta$-3 and $\beta-4$ as well as $\beta-6$ and $\beta-7$.

Table VIII lists also the charges and on the right hand side of picture 7 we see that the charge on the chloride atom converges with an increasing number of monomer units to an $\infty$ value of -0.823 for $\varnothing q_{1}$. This means that upon solvation the units are polarized such that the absolute value of the charge is increased by approximately $10 \%$.

\section{Energies}

In Table $\mathrm{X}$ we list the hydrogen bond energies for all clusters. The SEN hydrogen bond energy $f_{\mathrm{Cl}}$ yields one particular hydrogen bond energy between a donor and an acceptor. It is calculated according to the shared-electron number method developed by Reiher et al., ${ }^{45,51}$ see also Methodology section and Ref. 51.

From Table $\mathrm{X}$ and Fig. 8 we see that the hydrogen bond energy exhibits anticooperative effects. The strength of the individual as well as for the average hydrogen bond decreases with a larger cluster size. This trends are different from what was observed in chains of water molecules. ${ }^{63}$ It is in agreement with the observation from the previous sections, that the binding energy for the monomer exceeds that for the trimer and dimer.

We also calculated the binding energies $E_{I}$ of the chain complexes. Table XI lists the obtained values. Both binding energies per monomer units and per ions grow linearly in absolute values with the number of units. Considering the energies per units we find an increasing stability with an increasing number of units for the dissociation into monomer pairs.

The behavior of the energy per ions is depicted in Fig. 9. We find from dimer unit on an increasing stability. The monomer unit is more stable than the dimer or the trimer, as discussed in the previous sections. From the tetramer on the complexes are more stable than the monomer, which corresponds with the structural transition observed in the dipole moment. We also depicted the interaction energy of the most stable ring trimer structure. Only the nonamer is as stable as the trimer.

TABLE XI. Binding energies $E_{I}$ in $\mathrm{kJ} / \mathrm{mol}$. The first energy block considers the energies between all ions, whereas the second block considers dissociation into monomer pairs. $E_{I} / N o$ denotes the energies per number of unit.

\begin{tabular}{|c|c|c|c|c|c|c|c|c|c|c|}
\hline No. & $1 \mathrm{a}$ & $2 \mathrm{a}$ & $3 a$ & $4 a$ & $5 \mathrm{a}$ & $6 a$ & $7 \mathrm{a}$ & $8 \mathrm{a}$ & $9 \mathrm{a}$ & $\infty$ \\
\hline \multicolumn{11}{|c|}{ Ions } \\
\hline$E_{I}$ & -449.9 & -866.9 & -1329.9 & -1800.2 & -2270.9 & -2741.9 & -3212.5 & -3684.3 & -4156.5 & $\cdots$ \\
\hline$E_{I} / N o$ & -449.9 & -433.5 & -443.3 & -450.1 & -454.2 & -457.0 & -458.9 & -460.5 & -461.8 & -469.3 \\
\hline \multicolumn{11}{|c|}{ Monomer pair } \\
\hline$E_{I}$ & $\cdots$ & -49.4 & -117.0 & -190.4 & -265.9 & -342.7 & -419.6 & -498.0 & -576.2 & $\cdots$ \\
\hline$E_{I} / N o$ & $\cdots$ & -24.7 & -39.0 & -47.6 & -53.2 & -57.1 & -59.9 & -62.3 & -64.0 & -68.2 \\
\hline
\end{tabular}




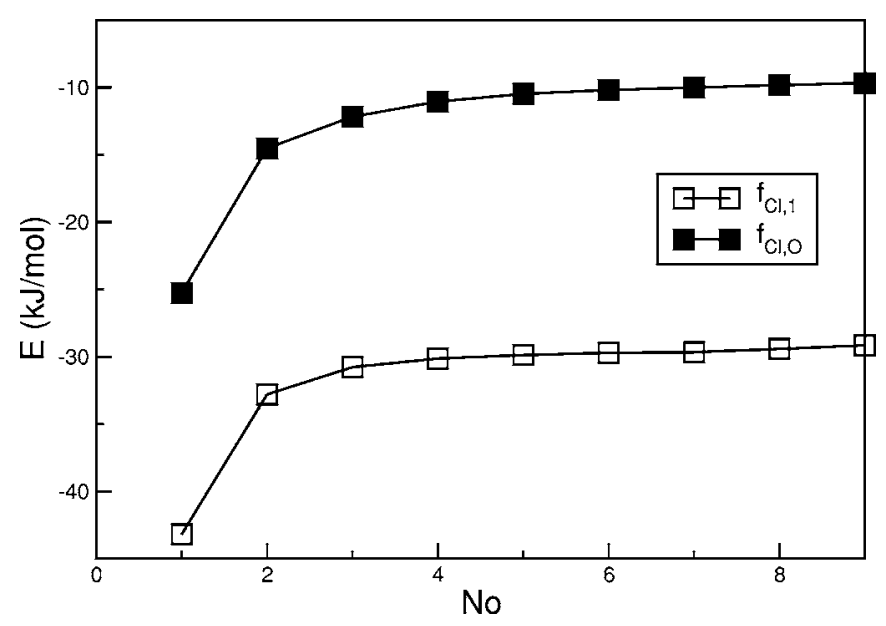

FIG. 8. SEN hydrogen bond energies plotted against the number of monomer units.

\section{DISCUSSION AND CONCLUSION}

The focus of this work is on the microscopic behavior of ionic liquids by means of static quantum chemical calculations. In order to gain deeper insight in the behavior of these liquids, we constructed higher clusters from monomer units that contain one cation and one anion. In the dimer elements the monomer units are buried in a very relaxed way, i.e., there is almost no difference between binding and reaction energy. We conclude that the monomer units are thus able to establish virtually ideal arrangements in the condensed phase. Ring structures seem to be very important. These structure motifs establish highly reduced dipole moments as compared to the analogous chain elements. This stands in accordance with polarity measurements that found polarities comparable to alcohols. ${ }^{59}$ Furthermore a comparison to radial pair distribution functions finds similar $\mathrm{C}-\mathrm{H} \cdots \mathrm{Cl}$ distances than the one established in the cyclic structure. The third point in favor of these structures is that they yield similar energies per monomer unit as the most stable monomer configuration treated within this work.

We also carried out a systematic study building up chains from one monomer unit. The $\mathrm{Cl} \cdots \mathrm{H}$ distance of the

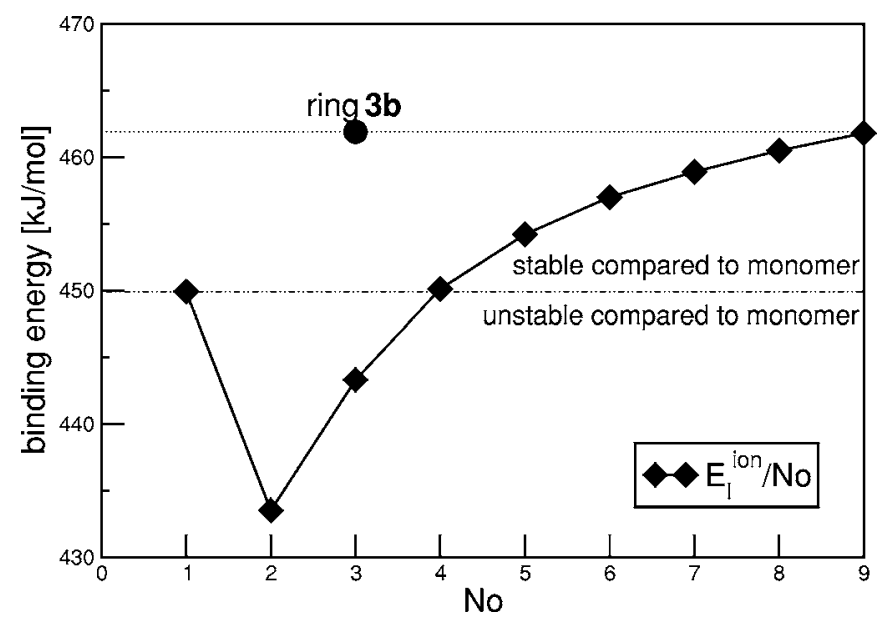

FIG. 9. Binding energies per ion plotted against the number of monomer units. chloride to the most acidic proton increases with an increasing number of monomer units. The value for the average bond grows asymptotically to $218.9 \mathrm{pm}$ which is again comparable to radial pair distribution functions. The growth of the difference to the monomer unit $\Delta r(\mathrm{Cl}-\mathrm{H})$ is larger than in water. The dipole moment grows almost linearly and the dipole moment per monomer unit reaches the asymptotic value of $16.3 \mathrm{D}$. The charges on the chloride atoms decrease with an increasing chain length. The extrapolation and behavior itself of charges with an increasing chain length are important in the light of molecular dynamics simulations. In molecular dynamics simulations atomic charges are needed to calculate an electrostatic term. In future a nice experiment would be to simulate a test reaction with standard and our extrapolated charges. Binding energies were obtained considering monomer pairs and ions. The energies grow linearly with an increasing number of units. Considering the ion binding energy per unit an increasing stability from the dimer is observed. While the dimer and the trimer are less stable than the monomer, from the tetramer on the aggregates are comparable in stability to the monomer unit.

\section{ACKNOWLEDGMENT}

Financial support by the collaborative research center SFB 624 "Templates" at the University of Bonn is gratefully acknowledged.

${ }^{1}$ T. Welton, Chem. Rev. (Washington, D.C.) 99, 2071 (1999).

${ }^{2}$ T. Welton, Coord. Chem. Rev. 248, 2459 (2004).

${ }^{3}$ Z. Liu, S. Haung, and W. Wang, J. Phys. Chem. B 108, 12978 (2004).

${ }^{4}$ J. Shah and E. Maginn, Fluid Phase Equilib. 222-223, 195 (2004).

${ }^{5}$ T. Morrow and E. Maginn, J. Phys. Chem. B 106, 12807 (2002).

${ }^{6}$ C. Margulis, H. Stern, and B. Berne, J. Phys. Chem. B 106, 12017 (2002).

${ }^{7}$ J. Lopes, J. Deschamps, and A. Padua, J. Phys. Chem. B 108, 2038 (2004).

${ }^{8}$ S. Urahata and M. Ribeiro, J. Chem. Phys. 120, 1855 (2004).

${ }^{9}$ T. Yan, C. Burnham, M. Del Pópolo, and G. Voth, J. Phys. Chem. B 108, 11877 (2004).

${ }^{10}$ S. Takahashi, K. Suzuya, S. Kohara, N. Koura, L. Curtiss, and M. Saboungi, Z. Phys. Chem. 209, 209 (1999).

${ }^{11}$ Z. Meng, A. Dolle, and W. Carper, J. Mol. Struct. 585, 119 (2002).

${ }^{12}$ A. Chaumont and G. Wipff, Inorg. Chem. 43, 5891 (2004).

${ }^{13}$ F. Gozzo, L. Santos, R. Augusti, C. Consorti, J. Dupont, and M. Eberlin, Chem.-Eur. J. 10, 6187 (2004).

${ }^{14}$ E. Talaty, S. Raja, V. Storhaug, A. Dolle, and W. Carper, J. Phys. Chem. B 108, 13177 (2004)

${ }^{15}$ Y. Paulechka, G. Kabo, A. Blokhin, A. Vydrov, J. Magee, and M. Frenkel, J. Chem. Eng. Data 48, 457 (2003).

${ }^{16}$ J. de Andrade, E. Boes, and H. Stassen, J. Phys. Chem. B 106, 13344 (2002).

${ }^{17}$ E. Turner, C. Pye, and R. Singer, J. Phys. Chem. A 107, 2277 (2003).

${ }^{18}$ Y. Wang, H. Li, and S. Han, J. Chem. Phys. 123, 174501 (2005).

${ }^{19}$ P. Hunt and I. Gould, J. Phys. Chem. A 110, 2269 (2006).

${ }^{20}$ C. G. Hanke, S. L. Price, and R. M. Lynden-Bell, Mol. Phys. 99, 801 (2001).

${ }^{21}$ C. G. Hanke, N. Atamas, and R. M. Lynden-Bell, Green Chem. 4, 107 (2002).

${ }^{22}$ R. M. Lynden-Bell, Mol. Phys. 101, 2625 (2003).

${ }^{23}$ T. Yan, C. J. Burham, M. G. Del Pópolo, and G. A. Voth, J. Phys. Chem. B 108, 11877 (2004).

${ }^{24}$ M. Bühl, A. Chaumont, R. Schurhammer, and G. Wipff, J. Phys. Chem. B 109, 18591 (2005).

${ }^{25}$ M. G. Del Pópolo, R. M. Lynden-Bell, and J. Kohanoff, J. Phys. Chem. B 109, 5895 (2005)

${ }^{26}$ B. L. Bhargava and S. Balasubramanian, Chem. Phys. Lett. 417, 486 (2006). 
${ }^{27}$ E. Ermakova, J. Solca, G. Steinebrunner, and H. Huber, Chem.-Eur. J. 4, 373 (1998).

${ }^{28}$ B. Kirchner, E. Ermakova, J. Solca, and H. Huber, Chem.-Eur. J. 4, 379 (1998).

${ }^{29}$ A. van der Avoird, E. H. T. Olthof, and P. E. S. Wormer, J. Chem. Phys. 105, 8034 (1996).

${ }^{30} \mathrm{~J}$. Hutter (private communication).

${ }^{31}$ P. Salvador, N. Kobko, R. Wieczorek, and J. J. Dannenberg, J. Am. Chem. Soc. 126, 14190 (2004).

${ }^{32}$ R. Wieczorek and J. J. Dannenberg, J. Am. Chem. Soc. 126, 14198 (2004).

${ }^{33}$ R. A. Klein, B. Mennucci, and J. Tomasi, J. Phys. Chem. A 108, 5851 (2004).

${ }^{34}$ B. Kirchner and M. Reiher, J. Am. Chem. Soc. 127, 8785 (2005).

${ }^{35}$ B. Kirchner, J. Chem. Phys. 123, 204116 (2005).

${ }^{36}$ R. Ahlrichs, M. Bär, M. Häser, H. Horn, and C. Kölmel, Chem. Phys. Lett. 162, 165 (1989); current version: see http://www.turbomole.de

${ }^{37}$ A. D. Becke, Phys. Rev. A 38, 3098 (1988).

${ }^{38}$ J. P. Perdew, Phys. Rev. B 33, 8822 (1986).

${ }^{39}$ A. D. Becke, J. Chem. Phys. 98, 5648 (1993).

${ }^{40}$ P. J. Stephens, F. J. Devlin, C. F. Chabalowski, and M. J. Frisch, J. Phys. Chem. 98, 11623 (1994).

${ }^{41}$ The tURBOMOLE basis set library is available via anonymous ftp from $\mathrm{ftp} / / / \mathrm{ftp}$. chemie.uni-karlsruhe.de/pub/basen

${ }^{42}$ D. E. Woon and T. H. Dunning, J. Chem. Phys. 100, 2975 (1993).

${ }^{43}$ S. F. Boys and F. Bernardi, Mol. Phys. 19, 553 (1970).

${ }^{44}$ F. B. van Duijneveldt, J. G. C. M. van Duijenveldt-van de Rijdt, and J. H. van Lenthe, Chem. Rev. (Washington, D.C.) 94, 1873 (1994).

${ }^{45}$ M. Reiher, D. Sellmann, and B. A. Hess, Theor. Chem. Acc. 106, 379 (2001).

${ }^{46}$ E. R. Davidson, J. Chem. Phys. 46, 3320 (1967).

${ }^{47}$ K. R. Roby, Mol. Phys. 27, 81 (1974).

${ }^{48}$ R. Heinzmann and R. Ahlrichs, Theor. Chim. Acta 42, 33 (1976).

${ }^{49}$ C. Ehrhardt and R. Ahlrichs, Theor. Chim. Acta 68, 231 (1985).

${ }^{50}$ M. Reiher and B. Kirchner, J. Phys. Chem. A 107, 4141 (2003).

${ }^{51}$ J. Thar and B. Kirchner, J. Phys. Chem. A 110, 4229 (2006) .

${ }^{52}$ G. Schaftenaar and J. H. Noordik, J. Comput.-Aided Mol. Des. 14, 123 (2000).

${ }^{53}$ S. Grimme, J. Comput. Chem. 25, 1463 (2004).

${ }^{54}$ S. Grimme, J. Phys. Chem. A 109, 3607 (2005).

${ }^{55}$ E. R. Johnson and A. D. Becke, J. Chem. Phys. 123, 024101 (2005).

${ }^{56}$ W. Koch and M. C. Holthausen, A Chemist's Guide to Density Functional Theory (Wiley-VCH, Weinheim, 2000).

${ }^{57}$ D. Martin and H. G. Hauthal, Dimethylsulfoxid (Akademie-Verlag, Berlin, 1971).

${ }^{58}$ C. Wakai, A. Oleinikova, M. Ott, and H. Weingärtner, J. Phys. Chem. B 109, 17028 (2005)

${ }^{59}$ C. Reichardt, Green Chem. 7, 339 (2005).

${ }^{60}$ A. J. Arduengo, H. V. R. Dias, R. L. Harlow, and M. Kline, J. Am. Chem. Soc. 114, 5530 (1992).

${ }^{61}$ K. N. Woods and H. Wiedemann, J. Chem. Phys. 123, 134506-1 (2005).

${ }^{62}$ C. Hardacre, J. D. Holbrey, S. E. J. McMath, D. T. Bowron, and A. K. Soper, J. Chem. Phys. 118, 273 (2003).

${ }^{63}$ T. Kar and S. Scheiner, J. Phys. Chem. A 108, 9161 (2004). 